\title{
Cross-Linking as an Adjuvant Treatment for Tectonic Corneal Lamellar Graft Preparation
}

\author{
George D. Kymionis ${ }^{*}$, , Argyro D. Plaka ${ }^{1}$, Vasilios F. Diakonis ${ }^{1}$, Michael A. Grentzelos ${ }^{1}$, \\ Georgios A. Kontadakis ${ }^{1}$, Andreana C. Mersinoglou ${ }^{2}$, Efstathios T. Detorakis ${ }^{1}$ and Fotis Topouzis ${ }^{2}$ \\ ${ }^{I}$ Vardinoyiannion Eye Institute of Crete (VEIC), Faculty of Medicine, University of Crete, Heraklion, Crete, Greece \\ ${ }^{2}$ Department of Ophthalmology, School of Medicine, Aristotle University of Thessaloniki, Thessaloniki, Greece
}

\begin{abstract}
Purpose: To describe a new surgical approach for the tectonic reconstruction of the anterior ocular segment and the management of scleral and conjuctival melting.

Methods: Case series of patients demonstrating anterior segment anomalies, such as scleral and conjunctival melting. The anterior stromal part of a pre-cut corneal graft for Descemet's stripping automated endothelial keratoplasty (DSAEK) was cross-linked with riboflavin and ultraviolet A irradiation and was used to cover scleral (scleral melting in a patient with necrotizing scleritis, one case) and conjuctival (dehiscence of the conjunctiva in patients with anti-glaucomatous valve exposure, two cases) areas. The endothelial part of all corneal grafts was used for DSAEK in patients with need of corneal endothelial transplantation.

Results: Repair of scleral and conjuctival melting was successful in all cases. No intra- or post- operative complications occured. Visual acuity of all patients remained stable during the follow up period. One year postoperatively the corneal graft remained in place and no signs of inflammation were evident, while all grafts were epithelialized.

Conclusions: The use of cross-linked corneal graft for the tectonic reconstruction of the anterior ocular segment represents an adequate surgical approach for the management of scleral and conjuctival melting; while, at the same time it offers the advantage of using one corneal graft for two recipients.
\end{abstract}

Keywords: Corneal collagen cross-linking, scleral melting, conjuctival melting, corneal transplantation.

\section{INTRODUCTION}

Anterior segment tectonic reconstruction in patients with scleral and conjuctival melting has been managed using various types of homografts and allografts as patching material [1-4]. Clinical situations that require such surgical interventions include anterior necrotizing scleritis [5], exposure of implant material employed in previous procedures (such as anti-glaucomatous valve tubes or plates [6], encircling bands for retinal detachment surgery [7] or intraorbital implants used in enucleation or evisceration surgeries [8]). Reinforcement of thinned sclera or covering of exposed implants is necessary, to prevent secondary infection and/or prolapse of ocular contents [5]. Moreover, in the case of anti-glaucomatous valves, the exposure of the valve tube or plate may result in loss of valve function [6]. A variety of patching materials has been used so far, including cadaveric fascia lata, dura, sclera, cornea, buccal mucosa or hard palate homografts as well as amniotic membrane grafts. The ideal material for this purpose should demonstrate high mechanical stability and rigidity, biocompatibility, lack of inflammatogenic activity and low cost.

*Address correspondence to this author at the University of Crete, Faculty of Medicine, Vardinoyiannion Eye Institute of Crete (VEIC), 71003 Heraklion, Crete, Greece; Tel: +302810371800; Fax: +302810394653;

E-mail: kymionis@med.uoc.gr
We present a novel surgical technique for the management of scleral and conjuctival melting with the use of the anterior cross-linked stromal part of a corneal graft for Descemet's stripping automated endothelial keratoplasty (DSAEK).

\section{MATERIALS AND METHODOLOGY}

\section{Case Series}

\section{Scleral Melting}

A 70-year-old female patient presented to our clinic complaining of severe and persistent pain at the orbital area of her left eye that did not respond to analgesic medication. She had undergone pterygium surgery followed by topical application of mitomycin C (MMC) 15 years ago. The uncorrected distance visual acuity (UDVA) was 20/80 at the affected eye. Slit-lamp examination revealed a 4 x $2.5 \mathrm{~mm}$ area of scleral melting nasally (Fig. 1a). Conjuctival scraping was performed and swabs were sent for culture.

\section{Conjuctival Melting}

Two male patients aged 72 and 75 year, respectively, were referred to our clinic for the management of Ahmed valve tube extrusion, concerning their right eyes. Both patients had undergone trabeculectomy twice in the past for the management of chronic open angle glaucoma. Patients 

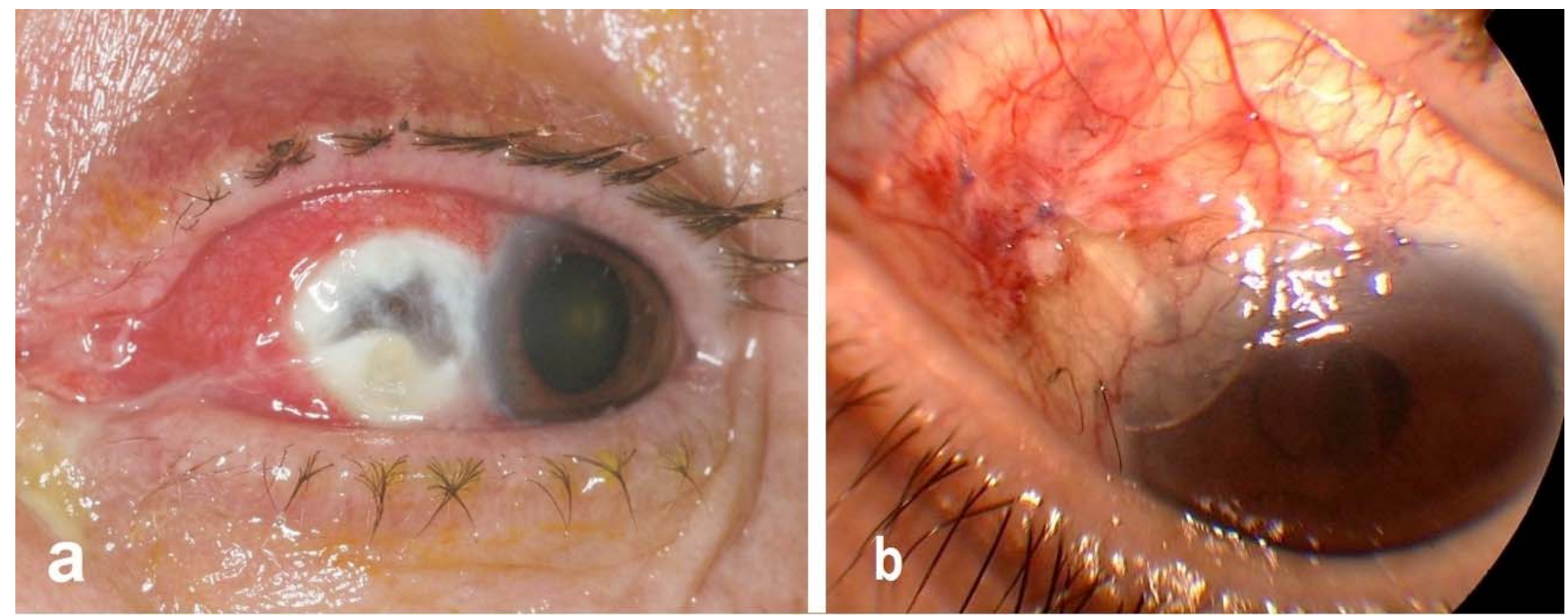

Fig. (1). Patient demonstrating scleral melting due to necrotizing scleritis, slitlamp image at presentation (a), image of patient demonstrating anti-glaucomatous valve tube extrusion (b).

presented with valve tube extrusion 3 years and 11 months after valve insertion (Fig. 1b). Corrected distance visual acuity (CDVA) was $20 / 25$ and counting fingers at $50 \mathrm{~cm}$ distance, respectively.

\section{Surgical Intervention}

All patients were informed of risks and benefits prior to surgical intervention, and they gave their written informed consent in accordance with institutional guidelines and the Declaration of Helsinki for human research. In all cases we decided to cover the area of the lesion with the anterior stroma harvested from a graft used at the same day for Descemet's stripping automated endothelial keratoplasty (DSAEK) on patients with corneal endotheliopathies (one patient with Fuchs' endothelial dystrophy and two patients with post-cataract bullous keratopathy). The anterior stromal cup from the DSAEK graft was crosslinked immediately after DSAEK with the use of riboflavin and ultraviolet A irradiation in accordance with the Dresden protocol described in previous studies [9-11].

The operation was performed under topical anesthesia (Alcaine drops, Alcon, Hellas and Xylocaine gel, AstraZeneca, Sweden) in all three cases. The corneal stromal graft was applied on the bare sclera at the region of necrosis (1 case) and at the area of the exposed valve tube (2 cases). In all cases the graft was stabilized on the surface of the sclera with 3 cardinal prolene sutures 5-0. Conjunctiva from the adjacent region was used to cover a portion of the corneal button and was fixed on its surface with vicryl 7-0 sutures. No signs of graft dehiscence or any other complication were noted immediately postoperatively in all cases.

One week after the procedure patients were discharged, topical ciprofloxacin (Exocin, Alvia, Greece) and artificial tears were administered. Within two weeks after treatment the corneal grafts were noted to be covered with epithelium. During the one year follow up period, the grafts remained in place and no signs of inflammation or graft melting rejection were evident, while all grafts were completely epithelialized (Fig. 2a, b). Visual acuity of all patients remained unchanged throughout the one year follow-up when compared with the preoperative values.

\section{DISCUSSION}

During the past decade a series of new technologies and new surgical treatments were introduced in respect with the cornea. The most important interventions are the partial corneal transplantations (anterior and posterior), corneal collagen cross linking for the treatment of corneal ectatic disorders and the development of femtosecond lasers. In our case series we take advantage of two of these new developments and present a new surgical approach for the tectonic reconstruction of the anterior ocular surface in cases of scleral and conjuctival melting.

Corneal collagen cross-linking has been proven to increase corneal rigidity more than 3 times after treatment [9]; moreover the avascular corneal tissue is free of keratocyte cells after CXL due to the induced apoptosis at the anterior 350 microns [12]. In our case series we used the anterior stroma from a graft used the same day for DSAEK (otherwise discarded); pachymetry of the grafts was between 330 and 360 micron (measuring only the stroma without the epithelium). The inserted graft was a reinforced avascular (minimizing the possibility of graft rejection due to autoimmune system activation) collagen button free of keratocytes, making this patching material strong, biocompatible and cost effective (one corneal graft being used for two recipient patients).

Different treatment approaches have been described for the tectonic reconstruction of the anterior ocular surface in case of scleral and conjunctival melting [13-15]. Patching is necessary to avoid possible infection, inflammation and antiglacomatous valve malfunction. In our case series this new surgical approach did not demonstrate any intra- or postoperative complications, such as graft melting - rejection and infection. Furthermore, visual acuity of all patients remained stable and unchanged throughout the one year follow-up and the graft was epithelialized completely 1 to 2 weeks after insertion. 

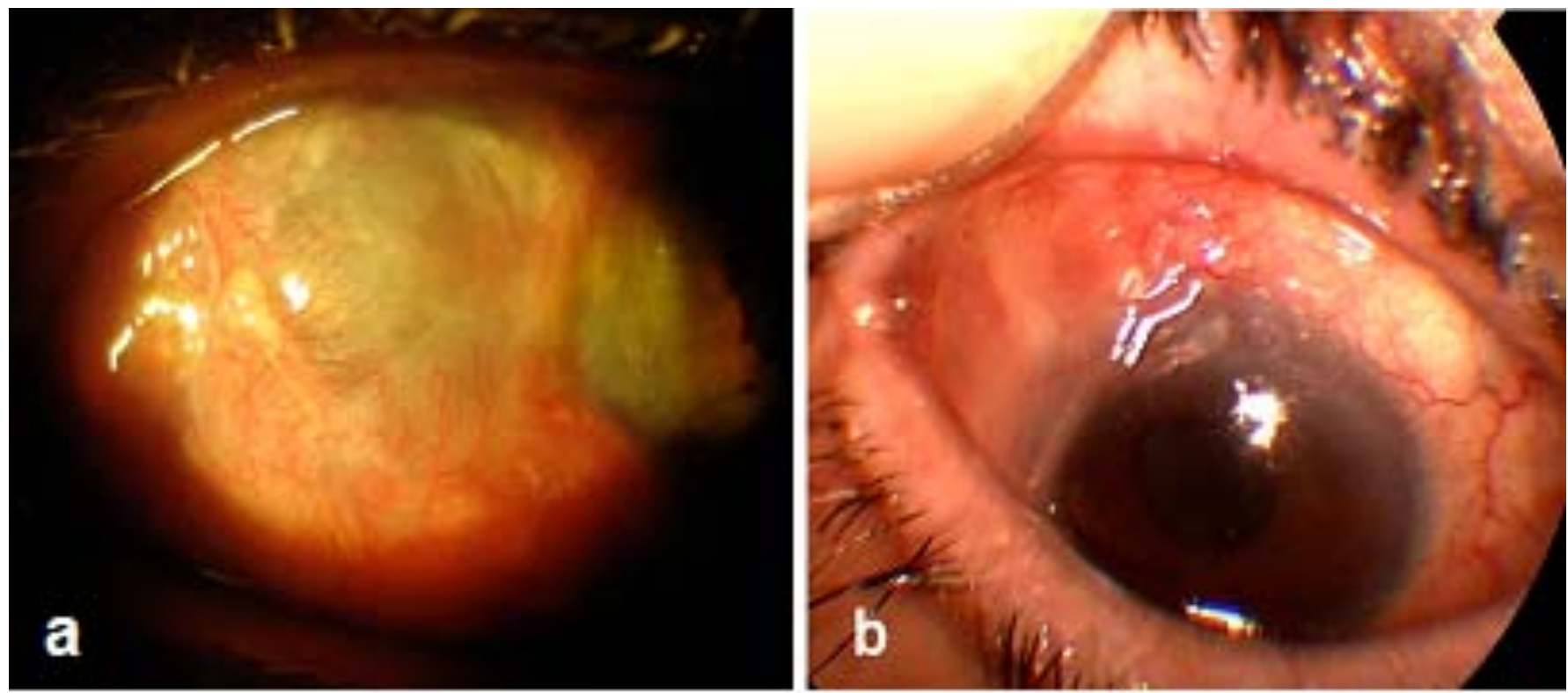

Fig. (2). Postoperative images demonstrating the grafts stability and epithiliazation (a) at the 1 year follow up of the patient with scleral melting and (b) at the six months follow up of the first patient with conjuctival melting.

Transparency of the cornea offers the ability of complete and detailed monitoring of the underlying tissues (sclera's healing process and revascularization) and implant material (valve tube). However it may not provide satisfactory aesthetical covering of the affected area. In order to improve the aesthetical result, a conjunctival flap may be used to cover the graft a couple of months after the initial procedure.

In conclusion, corneal patch consisting of the previously crosslinked anterior stromal cup from a DSAEK graft was effective in the management of scleral perforation. To confirm the efficacy and safety of the procedure a larger number of patients and longer follow-up is needed.

\section{CONFLICT OF INTEREST}

The authors confirm that this article content has no conflict of interest.

\section{ACKNOWLEDGEMENTS}

Declared none.

\section{REFERENCES}

[1] Esquenazi S. Autogenous lamellar scleral graft in the treatment of scleral melt after pterygium surgery. Graefes Arch Clin Exp Ophthalmol 2007; 245: 1869-71.

[2] $\mathrm{Ti}$ SE, Tan DT. Tectonic corneal lamellar grafting for severe scleral melting after pterygium surgery. Ophthalmology 2003; 110: 1126-36.

[3] Bene DJ. Treatment of scleral melt associated with uveal prolapse by pericardium grafting in Wegener's granulomatosis. Metab Pediatr Syst Ophthalmol 1999-2000; 22-23: 7-9.
[4] Tanji TM, Lundy DC, Minckler DS, Heuer DK, Varma R. Fascia lata patch graft in glaucoma tube surgery. Ophthalmology 1996; 103: 1309-12.

[5] Owoeye JF, Ayorinde OO. Surgically induced necrotising scleritis after pterygium excision. West Afr J Med 2012; 31: 142-4.

[6] Byun YS, Lee NY, Park CK. Risk factors of implant exposure outside the conjunctiva after Ahmed glaucoma valve implantation. Jpn J Ophthalmol 2009; 53: 114-9.

[7] Bernardino CR, Mihora LD, Fay AM, Rubin PA. Orbital complications of hydrogel scleral buckles. Ophthal Plast Reconstr Surg 2006; 22: 206-8.

[8] Detorakis ET, Ioannakis K, Drakonaki EE, Ganasouli D, Kozobolis VP. Processed fascia lata as an alternative implant material in evisceration. Ophthal Plast Reconstr Surg 2005; 21: 133-7.

[9] Wollensak G. Crosslinking treatment of progressive keratoconus: new hope. Curr Opin Ophthalmol 2006; 17: 356-360.

[10] Kymionis GD, Grentzelos MA, Kounis GA, Diakonis VF, Limnopoulou AN, Panagopoulou SI. Combined transepithelial phototherapeutic keratectomy and corneal collagen cross-linking for progressive keratoconus. Ophthalmology 2012; 119: 1777-84.

[11] Kymionis GD, Portaliou DM, Diakonis VF, Kounis GA, Panagopoulou SI, Grentzelos MA. Corneal collagen cross-linking with riboflavin and ultraviolet-A irradiation in patients with thin corneas. Am J Ophthalmol 2012; 153: 24-8.

[12] Kymionis GD, Diakonis VF, Kalyvianaki M, Portaliou D, Siganos C, Kozobolis VP, Pallikaris AI. One-year follow-up of corneal confocal microscopy after corneal cross-linking in patients with post laser in situ keratosmileusis ectasia and keratoconus. Am J Ophthalmol 2009; 147: 774-8

[13] Sangwan VS, Jain V, Gupta P. Structural and functional outcome of a scleral patch graft. Eye 2007; 21: 930-5.

[14] Muariello Jr JA, Pokorny K. Use of split thickness dermal grafts to repair corneal and scleral defects - a study of 10 patients. Br J Ophthalmol 1993; 77: 327-31.

[15] Ti S-E, Tan DTH. Tectonic corneal lamellar grafting for severe scleral melting after pterygium surgery. Ophthalmology 2003; 110: 1126-36.

This is an open access article licensed under the terms of the Creative Commons Attribution Non-Commercial License (http://creativecommons.org/licenses/by$\mathrm{nc} / 3.0 /$ ) which permits unrestricted, non-commercial use, distribution and reproduction in any medium, provided the work is properly cited. 